\title{
High resolution quantitative model-based HVAC fault detection and diagnosis considering building operation uncertainty
}

\author{
Yifu Shi, Godfried Augenbroe \\ Georgia Institute of Technology
}

\begin{abstract}
Along with the development of data analysis, the datadriven fault detection and diagnoses (FDD) method becomes very popular. However, the data-driven FDD methods requires a large amount of data from Building Automation System (BAS), and these data are usually unreliable. In this study, a co-simulated model which integrated the building energy model with HVAC detail model is developed in the co-simulated platform Building Controls Virtual Test Bed (BCVTB). The white box co-simulation model can provide reliable trending data to develop and validate the data-driven FDD algorithm. Since the deterministic model is not sufficient to reflect the actual operation conditions, the building physical and operational uncertainty is considered in this study by using Monte Carlo method. Typical AHU faults are configured in the model, and an FDD algorithm is developed and validated based on the specific building operation condition.
\end{abstract}

\section{Introduction}

Automated fault detection and diagnosis (FDD) in HVAC and other building system started from the late 1980s and early 1990s (Li, 2012). Katipamula and Brambley classified FDD methods by three types: process historybased method, qualitative model-based method and quantitative model-based method, which is shown in Figure 1 (Katipamula \& Brambley, 2005).

FDD studies associated with heating, ventilation air conditioning, and refrigeration (HVAC\&R) have increased in number since 2004. In 2017, Kim and Katipamula reviewed 197 studies conducted in the last two decades and found out that $62 \%$ of the FDD methods were process history-based, $26 \%$ were qualitative modelbased, and $12 \%$ were quantitative model-based (Kim \& Katipamula, 2017).

For the qualitative model-based methods, the biggest strength is that they are simple to develop and apply. However, qualitative model is typically specific to a specific system or a process, which makes it difficult to ensure that all rules are always applicable especially for complex systems. The rule-based methods, as the most popular qualitative model-based method, depends on the expertise and knowledge of developers (Katipamula \& Brambley, 2005) and generally have low diagnostic accuracies, especially at slight severity levels (Wang, et al., 2017).

Based on the statistical result, process history-data based methods has become the most popular research area and Principle Component Analysis (PCA) is the dominant algorithm among the process history-based methods. PCA has already been applied to AHU sensor fault diagnosis (Wang \& Xiao, 2004) (Xiao, Wang, \& Zhang, 2006), VAV system sensor fault diagnosis (Du, Jin, \& Wu, 2007), and chiller sensor fault diagnosis (Wang \& Cui, 2005). PCA-based FDD methods are based on the premise that anomalous sensor readings are very different from the normal data. Therefore, faults can be detected by finding sensor data that are "far away" from the median of the principal components of the sensor data. For fault diagnosis, however, PCA becomes less useful because of the non-causal relationship of the data. In the non-causal relationship, it is hard to identify whether changes in one variable will result in changes in another variable. Xiao et

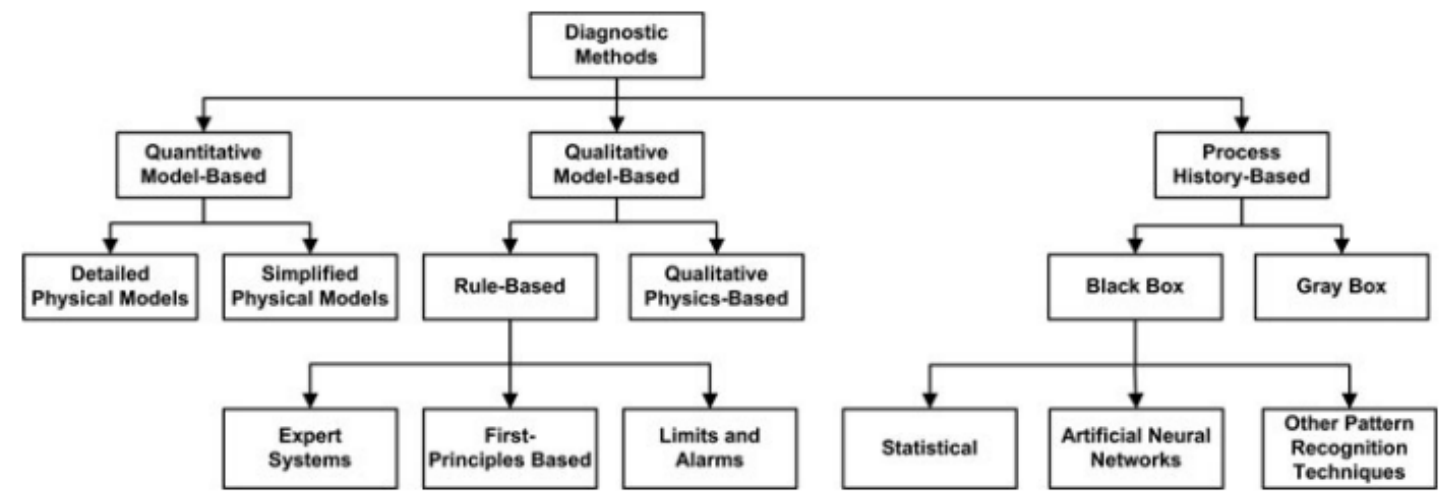

Figure 1: Classification scheme for AFDD method. 
al. proposed a PCA-based FDD method, however, the method was too weak to identify complex sensor faults because the sensor faults in the AHU are propagated to other parts of the system (Xiao, Wang, \& Zhang, 2006). Han et al. applied a support vector machine (SVM) to detect and diagnose chiller faults (Han, Cao, Gu, \& Ren, 2010), in which the FDD problem was viewed as a multiclass classification problem. Zhao et al. introduced a support vector data description (SVDD) for chiller FDD in which the FDD problem was transformed to a singleclass classification problem (Yang Zhao, 2013) (Zhao, Xiao, Wen, Lu, \& Wang, 2014). Yan et al. developed a semi-supervised learning, multi-class SVM as base classifier to classify faults (Yan, Zhong, Ji, \& Huang, 2018)

Process history-data based methods have no requirement of deep understanding of the system. However, the process history-data based methods require a long term to record the history data to train the non-fault and fault models. It is not only time consuming but also with two disadvantages. Firstly, it is difficult to obtain the history data with clear labelled operation status. For the measurements of fault-free conditions, it is claimed that the measurement data is easy to obtain and inexpensive, however, it is hard to guarantee that the measurement is coming from the pure fault-free condition, especially in the practical BAS operation conditions. For the measurement of fault conditions, however, the measured data is very expensive to be obtained and it is hard to link the data to its corresponding fault condition. If the polluted data is used to train the models, the accuracy of fault detection and diagnosis will be challenged. Secondly, all the history data is only recording the measurement for the actual occurred fault conditions which is hard to determine the length of data collecting period to guarantee the data including all major fault conditions of the equipment. Moreover, the possibility of fault occurrence is influenced by many factors, such as the age of system, the maintenance conditions and the health states of related systems. To bypass these disadvantages, many studies use the same data resource which comes from ASHRAE test (ASHRAE RP 1312 project) to validate their FDD algorithm.

The quantitative model-based method, on the other hand, is based on sound physical or engineering principles. The development of high-fidelity models based on detailed information of fault mechanism are complex and time consuming, the quantitative model provides the most accurate estimators of output when they are well formulated. The developers have the flexibility to develop and simulate the faults condition models based on the requirement.

To mimic the response of each components in a HVAC system to specific building operation scenarios, a Modelica-based HVAC quantitative model is developed. Traditionally, the quantitative model-based simulation has been deterministic and static. However, the uncertainties of building operation caused by the lack of full knowledge have impact on the performance of HVAC system in the practical situation. Therefore, to evaluate the impact of building operation uncertainties on fault detection and diagnosis, the Modelica-based HVAC quantitative model is integrated with a building energy model to develop a co-simulation model system.

\section{Method}

Building simulation is a computational procedure to subject a model form to a defined virtual experiment, and the virtual experiment is based on the input parameters. the lack of knowledge for building situation leads to inevitable uncertainties. The uncertainties can be classified into model formed uncertainty and parameter uncertainty. In this study, we focus on the parameter uncertainties. the most important contributors of the parameter uncertainties are associated with internal and external conditions over the course of virtual experiment, most notably occupant, operation and weather dynamics (Wang Q. , 2016).

The operational uncertainty mainly relates to the operational states of HVAC system, various factors potentially contribute to the discrepancy between ideal predicted and observed system, such as sensor drift, and damper/valve faults. Occupancy variable uncertainty is about the relationship and interaction between occupants and buildings. The thermal comfort requirement of occupants and their behaviour, such as the operation of lighting and appliance have impact to the performance of HVAC system. Weather uncertainty relates the microclimate effort caused by the effect of urban heat island. the weather uncertainty reflects the variation of the external condition of building and HVAC system.

In the co-simulation model, the occupancy variability and weather uncertainty are considered in the building energy model and the system operational uncertainty is presented as discrete fault conditions in the AHU model

\section{Building Energy Model}

In the study, EnergyPlus is the major simulation tool to develop the building energy model. Occupant, lighting and appliance power densities, infiltration rate and weather conditions are pre-defined and quantified as the uncertainty input parameters of the building energy model. We use the Georgia Tech Uncertainty and Risk Analysis Workbench (GURA-W) to propagate the uncertainties. The process of uncertainty propagation is shown in Figure 2.

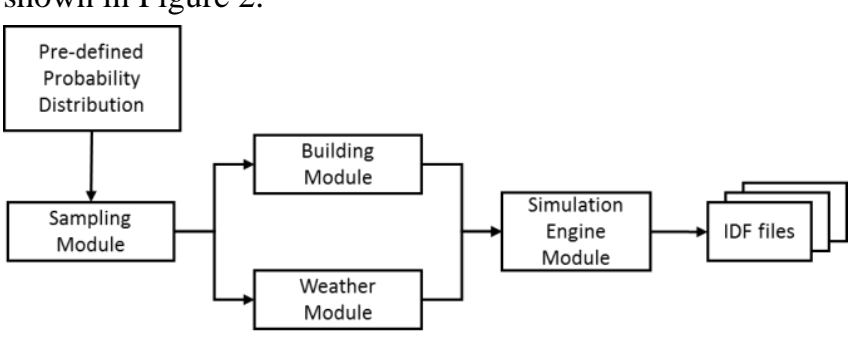

Figure 2: Process of uncertainty propagation.

We define the distributions of the uncertainty variables and store parameters of the probabilistic distribution in an XML file. The sampling module uses Latin Hypercube sampling to generate samples by using the pre-defined distribution parameters of each uncertain variable. In this 
study, typical meteorological year (TMY) file is used in weather module. The building module parses uncertainty variables in the EnergyPlus input files by searching occurrences of particular identifier tags and exposing these variables for further uncertainty analysis. The connection between sampling module and building module denotes an automated process that feeds Latin hypercube samples of uncertain variables to generate the input idf file for building energy model. The connection between sampling, weather and simulation engine module can generate weather files with the uncertainties with microclimate variables. (Wang Q. , 2016)

In this study, we developed a one-story office building model with 1000 square meter floor area and 3-meter height. The windows are located on the east and west walls with a height of 1 meter. The building is divided into two zones and served by an AHU system with two VAV boxes. The geometric scheme of the building is shown in Figure 3.

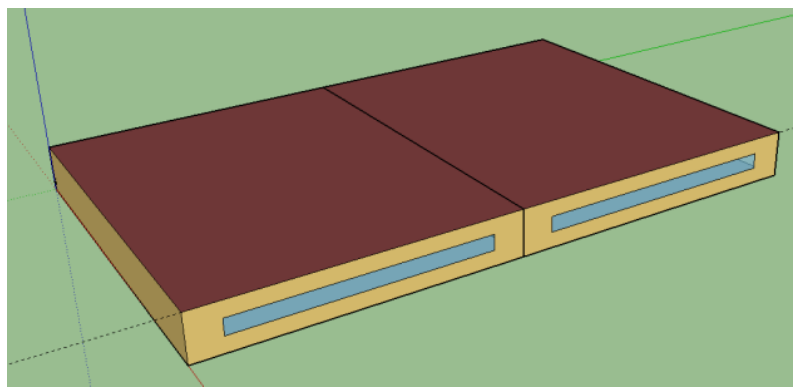

Figure 3: the Geometric scheme of the office building.

The building is located in Atlanta, in Climate Zone A3, the operation schedule and building construction information is based on the standard of ASHRAE reference building. To reflect the uncertainties of the building operation, the occupant density, lighting density, appliance power density and the infiltration rate are assumed to follow Gaussian distribution under two standard deviation levels and the parameters are defined as shown in Table 1. (The $20 \%$ scenario is normal distributed people, lighting, appliance power densities with a $20 \%$ standard deviation and normal distributed infiltration rate with a $10 \%$ standard deviation)

Table 1: parameter setting of selected variables.

\begin{tabular}{|c|c|c|c|}
\hline & $\begin{array}{l}\text { Mean } \\
\text { Value }\end{array}$ & $\begin{array}{c}\text { Standard } \\
\text { Deviation of } \\
10 \% \\
\text { scenarios } \\
\end{array}$ & $\begin{array}{c}\text { Standard } \\
\text { Deviation of } \\
20 \% \\
\text { scenarios } \\
\end{array}$ \\
\hline $\begin{array}{l}\text { Occupant } \\
\text { Density }\end{array}$ & $\begin{array}{c}15 \\
\mathrm{~m}^{2} / \text { person }\end{array}$ & $\begin{array}{c}1.5 \\
\mathrm{~m}^{2} / \text { person }\end{array}$ & $3 \mathrm{~m}^{2} /$ person \\
\hline $\begin{array}{l}\text { Lighting } \\
\text { Density }\end{array}$ & $15 \mathrm{~W} / \mathrm{m}^{2}$ & $1.5 \mathrm{~W} / \mathrm{m}^{2}$ & $3 \mathrm{~W} / \mathrm{m}^{2}$ \\
\hline $\begin{array}{l}\text { Appliance } \\
\text { Power } \\
\text { Density }\end{array}$ & $15 \mathrm{~W} / \mathrm{m}^{2}$ & $1.5 \mathrm{~W} / \mathrm{m}^{2}$ & $3 \mathrm{~W} / \mathrm{m}^{2}$ \\
\hline $\begin{array}{c}\text { Infiltration } \\
\text { Rate }\end{array}$ & $0.5 \mathrm{ACH}$ & $0.05 \mathrm{ACH}$ & $0.05 \mathrm{ACH}$ \\
\hline
\end{tabular}

100 energy model input idf files are generated, half of them are sampled with $10 \%$ deviation, and the other half are sampled with $20 \%$ deviation. The corresponding weather files based on the pre-determined probability distributions. The simulation period is 5 days from July $20^{\text {th }}$ to July $24^{\text {th }}$. To eliminate the simulation initialization stage, the first two days are treated as the initial period and the data during that period will not be used in the future analysis.

\section{HVAC System Model}

A Modelica-based single duct VAV system model is developed in Dymola. The air handling unit equips a supply fan; a mixing box with linked outside air damper, return air dampers and exhaust air damper; a cooling coil with dehumidify capacity; and a heating coil. The VAV box equips an air damper and a reheat coil. The water flow rate of the coil is maintained by a continuous adjustable water valve. And the air flow rate is maintained by the corresponding continuous adjustable air damper. The outside air temperature, mixed air temperature, supply air temperature and return air temperature are measured by temperature sensors. The static pressure and supply air flow are measured by pressure sensor and flow rate sensor respectively. The scheme of the system is shown in Figure 4 and the detailed system model in Dymola is shown in Figure 23.

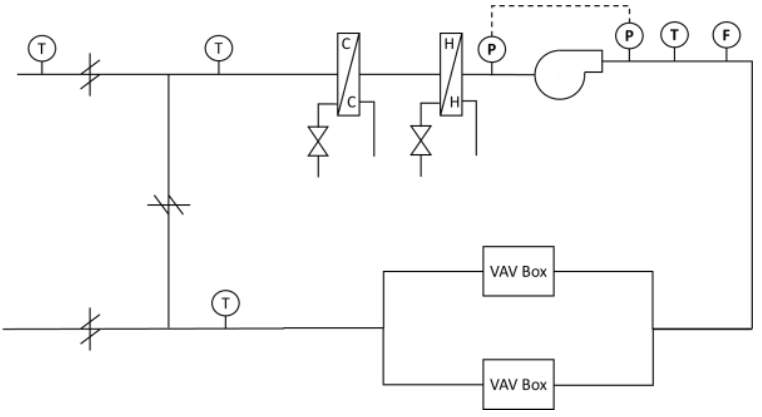

Figure 4: Scheme of HVAC model.

the HVAC system operates in the cooling mode since the simulation period is in July, and the system control strategy is shown below:

1. The two VAV boxes will maintain the space air temperature at cooling setpoint as $22^{\circ} \mathrm{C}$ from 6 am to $10 \mathrm{pm}$ and $26^{\circ} \mathrm{C}$ from $10 \mathrm{pm}$ to $6 \mathrm{am}$ by adjusting the air damper position. The

2. The supply fan adjusts the fan speed to maintain the static pressure setpoint.

3. The chilled water valve on cooling coil adjusts its position to maintain the supply air temperature setpoint as $15^{\circ} \mathrm{C}$.

4. The mixing box maintains the mixed air temperature setpoint at $17^{\circ} \mathrm{C}$ by adjusting the linked outside air damper, return air damper and exhaust air damper.

In this study, four fault conditions as well as the normal operation models are developed. The four fault conditions as shown below:

Fault scenario 1: outside damper cannot fully close, the minimum position is $50 \%$ open

Fault scenario 2: chilled water valve cannot fully open, the maximum position is $80 \%$ open

Fault scenario 3: chilled water valve cannot fully open, the maximum position is $60 \%$ open 
Fault scenario 4: supply air temperature reading has a $+2^{\circ} \mathrm{C}$ bias.

\section{Co-simulation Model}

With the combination of building energy model and HVAC system model, we can generate the building operation scenarios under different load related input parameters as well as the HVAC system operation states.

$$
\left.\begin{array}{l}
\operatorname{Bldg}\left(O_{i}, L_{i}, P_{i}, W_{i}\right) \\
\operatorname{System}\left(F_{j}\right)
\end{array}\right\} \operatorname{Scenarios}\left(O_{i}, L_{i}, P_{i}, W_{i}, F_{j}\right)
$$

In which, bldg. $\left(O_{i}, L_{i}, P_{i}, W_{i}\right)$ indicates the building load condition with the information of sampled uncertainty variables: Occupancy density $(\mathrm{O})$, Lighting density (L), Appliance Power density (P), Weather data (W). System $\left(F_{j}\right)$ indicates the HVAC operation states under the predefined fault $(\mathrm{F})$ condition.

The building energy model and HVAC model are integrated in Building Controls Virtual Test Bed (BCVTB), which is a simulation platform developed by Lawrence Berkeley National Lab. The building energy model and AHU system model communicate to each other at each simulation time step and the time step of the simulation is 1 minute. The energy model delivers the space condition measurements, such as space temperature and relative humidity to the HVAC model and the HVAC model sends back the delivered cooling/heating energy to the building energy model. The structure of the integrated model is shown in Figure 5.

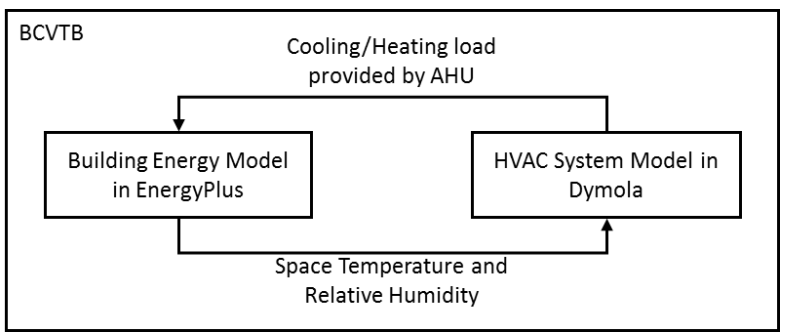

Figure 5: the structure of integrated model.

From the co-simulation, a multivariate vector $\boldsymbol{X}$ is composed by selected output variables as measurement observation at every time step. The variables include sensor readings, actuator states and controller setpoints. All the selected variables are measurable in the practical BAS system. A measurement sample is composed by all the observations for a specific building operation scenario during the simulation period. The co-simulated model in BCVTB is shown in Figure 6.

Based on the co-simulation model, a database contains the measurement samples for all building operation scenarios under specific uncertainty level.

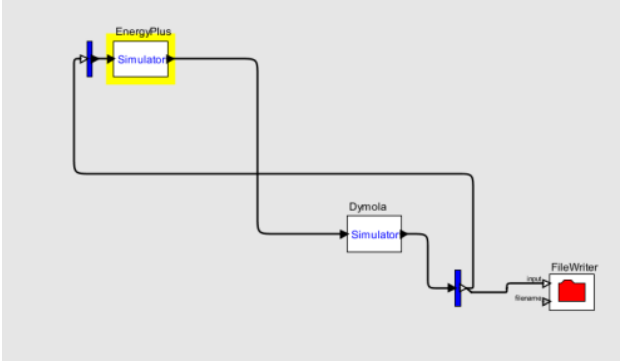

Figure 6: Integrated Model in BCVTB.

In the simulation, the observation contains 23 monitored variables and the descriptions of the selected variables are shown in Table 2.

Table 2: selected variables and the description.

\begin{tabular}{ll}
\hline Designation & Description \\
\hline OAF & Outside air flow \\
OADSP & Outside air flow setpoint \\
OAD & Outside air damper position \\
CHWVSP & Chilled water valve position setpoint \\
CHWV & Chilled water valve position \\
HWVSP & hot water valve position setpoint \\
HWV & hot water valve position \\
FanSSP & Fan speed setpoint \\
FanS & Fan speed \\
SAT & Supply air temperature \\
SAFT & Supply air total flow \\
SAF1 & Supply air flow for zone 1 \\
VAVD1 & VAV damper position for zone 1 \\
SAF2 & Supply air flow for zone 2 \\
VAVD2 & VAV damper position for zone 2 \\
RMT1 & Room temperature of zone 1 \\
RMT2 & Room temperature of zone 2 \\
RMRH1 & Room relative humidity of zone 1 \\
RMRH2 & Room relative humidity of zone 2 \\
RAT & Return air temperature \\
OAT & Outside air temperature \\
MAT & Mixed air temperature \\
\hline FOr each uncertainty deviation level (10\% and $20 \%), b y$
\end{tabular}

For each uncertainty deviation level (10\% and 20\%), by integrating the building energy model with 50 occupancy variability and weather uncertainty scenarios and the HVAC system model with 4 fault condition scenarios and a fault free scenario. Therefore, 110 samples are generated, and each sample contains 4320 observations. Among the 110 samples, 50 samples are simulated under normal operation and 15 samples are obtained under each fault condition. 50 samples of normal operation are randomly split into 40 and 10 samples for training and test. For each fault, 15 samples are randomly split into 10 and 5 samples for training and test. There are totally 80 samples in training set and 30 samples in test set for each uncertainty deviation level.

\section{FDD algorithm}

An FDD problem can be transformed into a classification problem. In order to distinguish the data of one fault class from other fault classes, we attempt to analyse the classification problem from data clustering point of view by measuring the distance between the samples.

If the samples of two different fault conditions are far away to each other, the two fault conditions are easily distinguished. However, if the distance between the samples from two fault conditions are small, it will 
increase the possibility to obtain false alarm or missing alarm.

When a new data comes, the distance between the new data multivariate observation vector $\boldsymbol{x}$ and the trained data multivariate vector $\boldsymbol{X}$ of each scenario combination will be calculated by Eq. (1)

$$
d_{i}=\left\|\boldsymbol{x}-\boldsymbol{X}_{\boldsymbol{i}}\right\|^{2},(i=1,2, \ldots, k)(1)
$$

the 10 smallest distances will be selected, and its corresponding operation scenarios will be selected as the candidate for the potential operation conditions of the new data sample. If most candidates are coming from the same fault condition $\mathrm{F}_{\mathrm{i}}$, the operation condition of the tested new data will be diagnosed as $F_{i}$. If the candidates are evenly located in different clusters which indicates that the algorithm cannot diagnose the operation condition of tested new data.

\section{Result}

\section{Simulation Result}

For each uncertainty deviation level, 40 samples under fault free condition are trained to develop the model for normal operation. Figure 7 to Figure 16 show the variation of selected variables of the 40 samples under $10 \%$ and $20 \%$ uncertainty levels. The red line is the mean value of each variable, the yellow line and the blue line are the 90 percentile and 10 percentile respectively.

Based on the results shown in figures, the co-simulated model can capture the responses of each components in HVAC system and provides the air conditions. The trend data reflect the impact of the uncertainties of building usage. Along with the increase of uncertainty, the chilled water valve and terminal air damper has a larger operating range to maintain the supply air temperature and space temperature respectively.

The comparison of return air temperature:

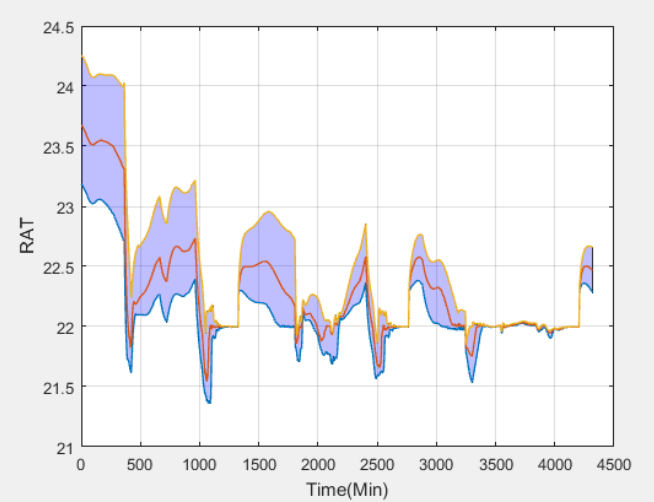

Figure 7: Return air temperature trend for normal operation under $10 \%$ uncertainty.

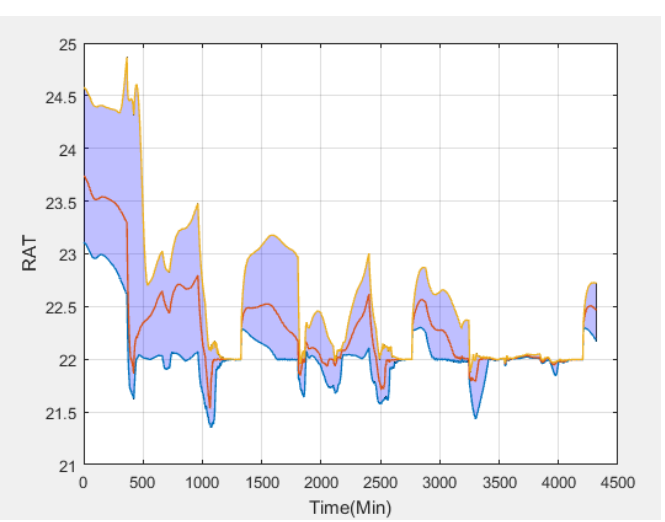

Figure 8: Return air temperature trend for normal operation under $20 \%$ uncertainty.

The comparison of chilled water valve position

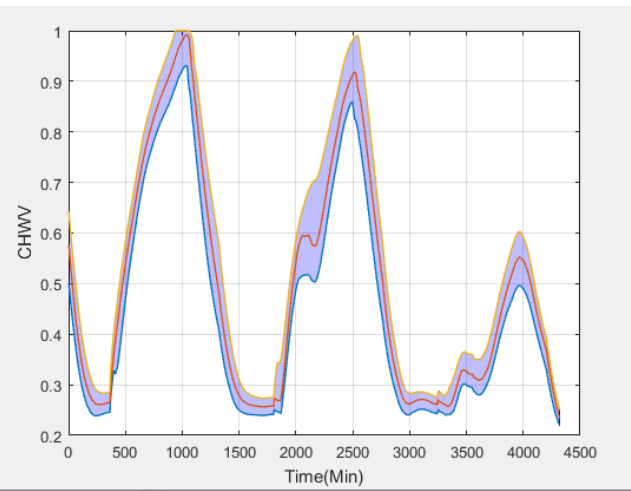

Figure 9: Chilled water valve position trend for normal operation under $10 \%$ uncertainty.

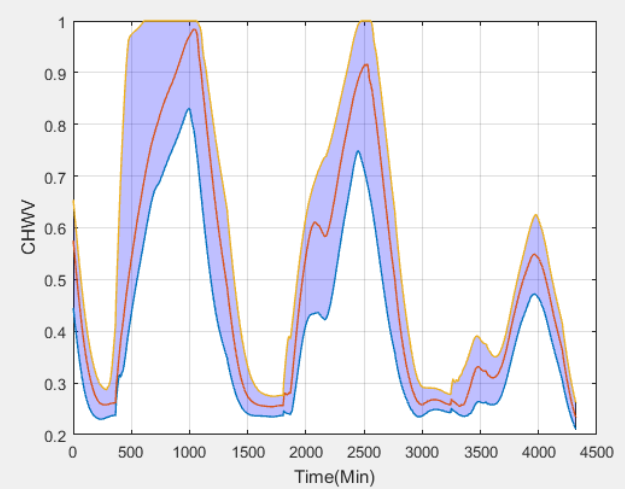

Figure 10: Chilled water valve position trend for normal operation under $20 \%$ uncertainty.

The comparison of fan speed 


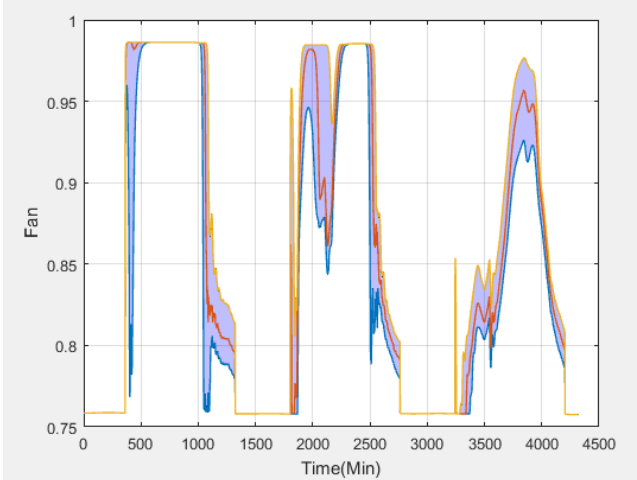

Figure 11: Fan speed trend for normal operation under $10 \%$ uncertainty.

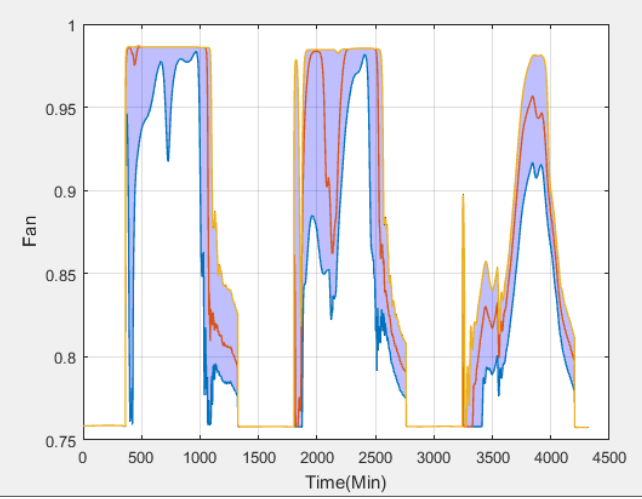

Figure 12: Fan speed trend for normal operation under $20 \%$ uncertainty.

The comparison of supply air temperature

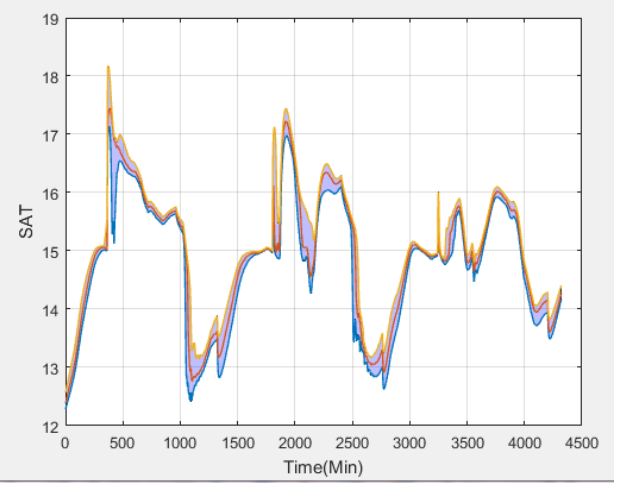

Figure 13: Supply air temperature trend for normal operation under $10 \%$ uncertainty.

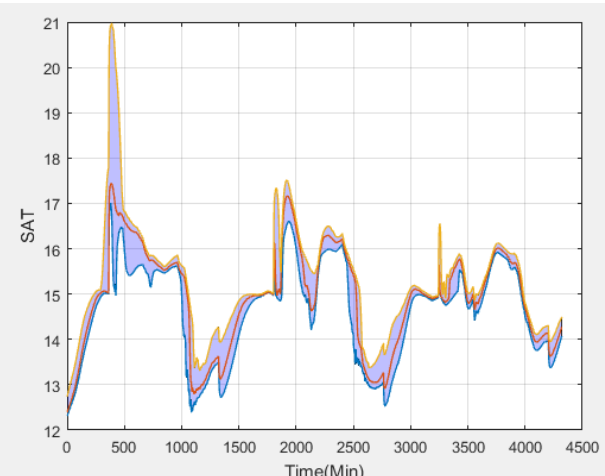

Figure 14 Supply air temperature trend for normal operation under $20 \%$ uncertainty
The comparison of VAV box damper position

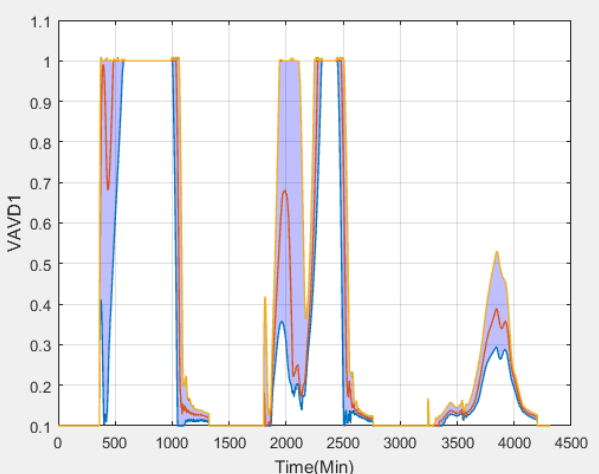

Figure 15: VAV damper of zone 1 trend for normal operation under $10 \%$ uncertainty.

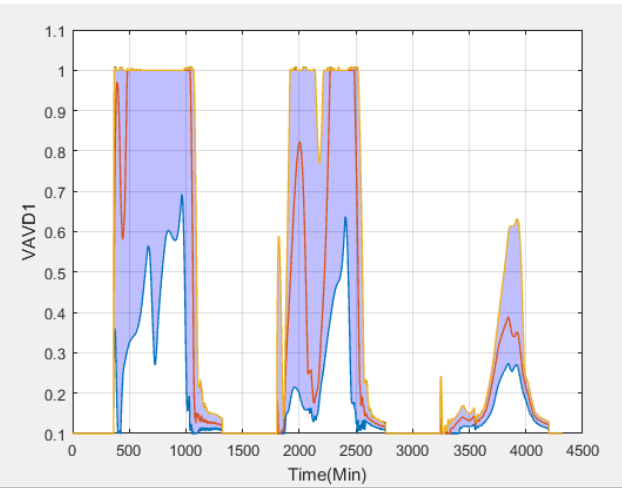

Figure 16: VAV damper of zone 1 trend for normal operation under $20 \%$ uncertainty.

\section{Fault Detection and Diagnosis Result}

By using the database generated by the co-simulation, the above-mentioned FDD algorithm is developed and tested. The FDD method are tested by using the randomly selected samples in the test set of normal operation and each fault conditions. The result of FDD under $10 \%$ and $20 \%$ uncertainty levels are shown in Table 3 and

\begin{tabular}{|c|c|c|c|c|c|}
\hline \multirow[b]{2}{*}{$\begin{array}{l}\text { True } \\
\text { Fault }\end{array}$} & \multicolumn{5}{|c|}{ Predicted candidate fault } \\
\hline & $\begin{array}{l}\text { Fault } \\
\text { free }\end{array}$ & $\begin{array}{c}\text { Fault } \\
1\end{array}$ & $\begin{array}{c}\text { Fault } \\
2\end{array}$ & $\begin{array}{c}\text { Fault } \\
3\end{array}$ & $\begin{array}{c}\text { Fault } \\
4\end{array}$ \\
\hline $\begin{array}{l}\text { No- } \\
\text { fault }\end{array}$ & $96 \%$ & 0 & $4 \%$ & 0 & 0 \\
\hline Fault 1 & 0 & $100 \%$ & 0 & 0 & 0 \\
\hline Fault 2 & $60 \%$ & 0 & $40 \%$ & 0 & 0 \\
\hline Fault 3 & 0 & 0 & $22 \%$ & $78 \%$ & 0 \\
\hline Fault 4 & $6 \%$ & 0 & 0 & 0 & $94 \%$ \\
\hline
\end{tabular}

Table 4 , respectively.

Table 3: FDD result of $10 \%$ variation scenarios.

\begin{tabular}{cccccc}
\hline \multirow{2}{*}{$\begin{array}{c}\text { True } \\
\text { Fault }\end{array}$} & \multicolumn{5}{c}{ Predicted candidate fault } \\
\cline { 2 - 6 } & $\begin{array}{c}\text { fault } \\
\text { free }\end{array}$ & $\begin{array}{c}\text { Fault } \\
1\end{array}$ & $\begin{array}{c}\text { Fault } \\
2\end{array}$ & $\begin{array}{c}\text { Fault } \\
3\end{array}$ & $\begin{array}{c}\text { Fault } \\
4\end{array}$ \\
\hline No- & $96 \%$ & 0 & $4 \%$ & 0 & 0 \\
fault & & & & 0 & 0 \\
Fault 1 & 0 & $100 \%$ & 0 & 0 & 0 \\
Fault 2 & $60 \%$ & 0 & $40 \%$ & 0 & 0 \\
Fault 3 & 0 & 0 & $22 \%$ & $78 \%$ & 0 \\
Fault 4 & $6 \%$ & 0 & 0 & 0 & $94 \%$ \\
\hline
\end{tabular}

Table 4: FDD result of $20 \%$ variation scenarios. 


\begin{tabular}{|c|c|c|c|c|c|}
\hline \multirow[b]{2}{*}{$\begin{array}{l}\text { True } \\
\text { Fault }\end{array}$} & \multicolumn{5}{|c|}{ Predicted candidate fault } \\
\hline & $\begin{array}{l}\begin{array}{l}\text { Fault } \\
\text { free }\end{array} \\
\text { f }\end{array}$ & $\begin{array}{c}\text { Fault } \\
1\end{array}$ & $\begin{array}{c}\text { Fault } \\
2\end{array}$ & $\begin{array}{c}\text { Fault } \\
3\end{array}$ & $\begin{array}{c}\text { Fault } \\
4\end{array}$ \\
\hline $\begin{array}{l}\text { No- } \\
\text { fault }\end{array}$ & $92 \%$ & 0 & 1 & 0 & 0 \\
\hline Fault 1 & 0 & $100 \%$ & 0 & 0 & 0 \\
\hline Fault 2 & $76 \%$ & 0 & $22 \%$ & $2 \%$ & 0 \\
\hline Fault 3 & $6 \%$ & 0 & $44 \%$ & $44 \%$ & $6 \%$ \\
\hline Fault 4 & $12 \%$ & 0 & $14 \%$ & 0 & $74 \%$ \\
\hline
\end{tabular}

From the result above, we can find that the FDD method can effectively diagnose fault 1 , fault 3 and fault 4 from the normal operation condition under $10 \%$ uncertainty level. But the method is not sensitive to distinguish the sample of fault 2 from the samples of normal operation, which will lead to the missing alarm. For the FDD result under $20 \%$ uncertainty level, we can find that the FDD method can still effectively diagnose Fault 1 from normal operation, however, the diagnostic accuracies for fault 3 and fault 4 is reduced, especially for fault 3. Comparing the results under $10 \%$ and $20 \%$ uncertainty levels, we can see that the method become less sensitive to the severity level, which make the system have a higher probability to mistakenly predict fault 2 as normal operation, as well as underestimate the severity level of the fault 3 , even though the method can still locate the fault 3 to the chilled water valve.

To analyse the results, the average internal distance between samples in same cluster and the average external distance from the samples in one cluster to the samples in other clusters are calculated by Eq (2) and Eq (3).

$$
\begin{array}{r}
\mathrm{d}_{\text {ave_int }}=\frac{1}{C_{n}^{2}} \sum\left\|\boldsymbol{X}_{\boldsymbol{i}}-\boldsymbol{X}_{\boldsymbol{j}}\right\|^{2},(i, j=1,2, \ldots, n ; i \neq j) \\
\mathrm{d}_{\text {ave_ext }}=\frac{1}{n m} \sum\left\|\boldsymbol{X}_{\boldsymbol{i}}-\boldsymbol{X}_{\boldsymbol{j}}\right\|^{2},(i=1,2, \ldots, n \text { and } j= \\
1,2, \ldots, m)
\end{array}
$$

The calculation results for $10 \%$ uncertainty level are shown from Figure 17 to Figure 21.

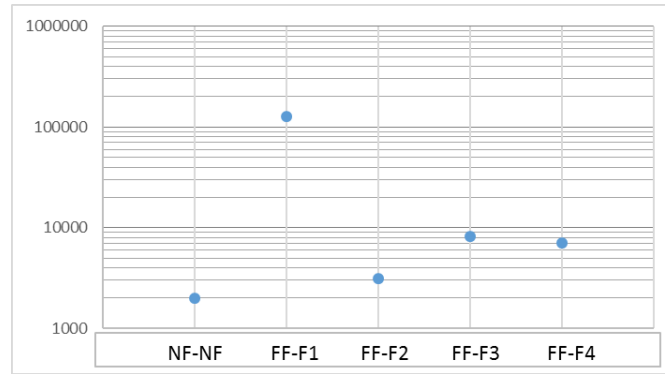

Figure 17: Average distance from Non-Fault condition under $10 \%$ uncertainty level.

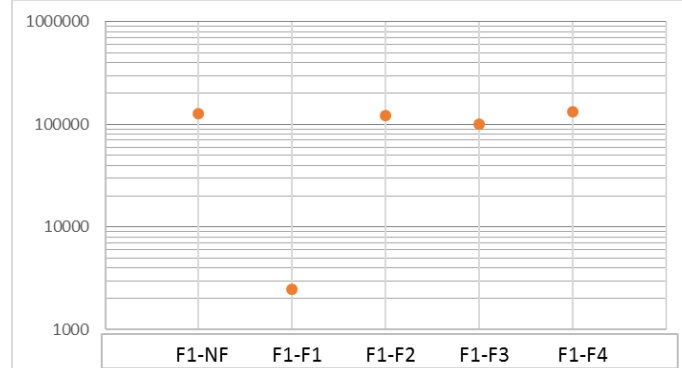

Figure 18: Average distance from Fault-1 condition under $10 \%$ uncertainty level.

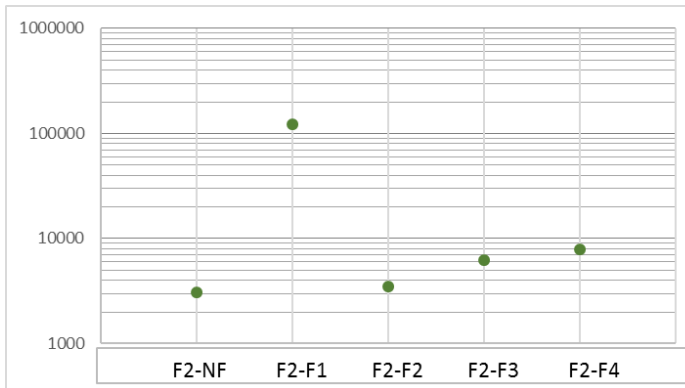

Figure 19: Average distance from Fault-2 condition under $10 \%$ uncertainty level.

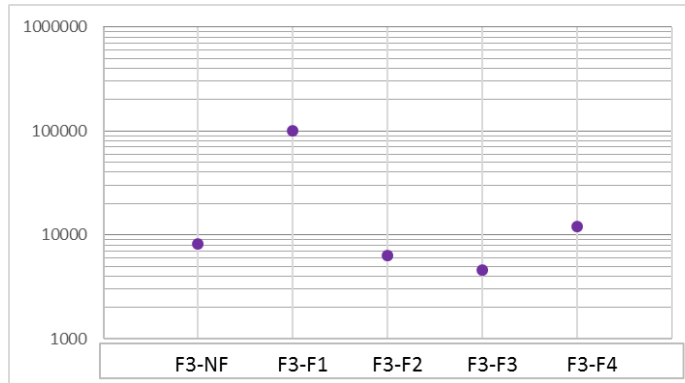

Figure 20: Average distance from Fault-3 condition under $10 \%$ uncertainty level.

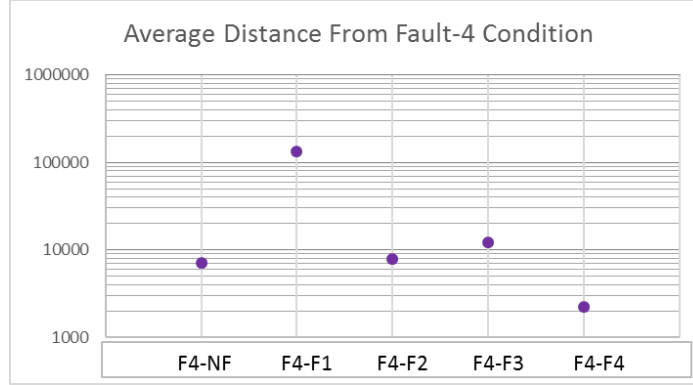

Figure 21: Average distance from Fault-4 condition under $10 \%$ uncertainty level.

Based on the result, the average internal distance of normal operation, fault 1 , fault 3 and fault 4 has relatively significant difference to the external distances to other clusters. It indicates that the fault condition is the main factor to shape the corresponding data sample. However, the average internal distance of fault 2 is similar to the external distance between fault 2 and the normal operation. It explains the reason why the algorithm cannot diagnose fault 2 from the normal operation. The real measured data also reflects the situation. We can see that the chilled water valve rarely moves to a position over $80 \%$ open in the normal condition from Figure 9, 
Therefore, even the chilled water valve cannot reach over $80 \%$ in fault 2 condition, the difference between the normal operation and faulty operation is very slight.

For the result shown in Figure 22 which is the average distance from fault 3 under $20 \%$ uncertainty level, we can find that the average internal distance of fault 3 becomes closer to the external distance between fault 3 and fault 2 along with the increasing uncertainty level. That is the reason why the method cannot distinguish the samples of fault 3 from the samples of fault 2 .

This comparison shows that the performance of an FDD algorithm may have different performance under different building usage condition. Therefore, the validation of an FDD algorithm based on a single standard usage database is not generic enough to evaluate the FDD algorithm.

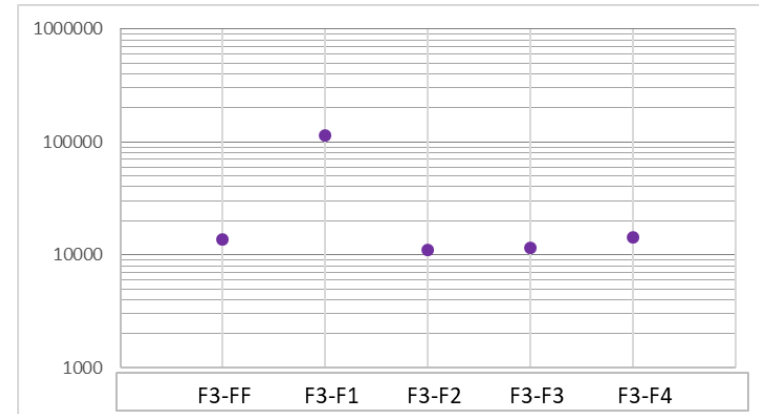

Figure 22: Average distance from Fault-3 condition under $20 \%$ uncertainty level.

\section{Conclusion}

In this study, a co-simulation model is developed which integrating building energy model and Modelica-based AHU system model. The model considers the variation and uncertainties of building usage and is able to capture the response of the components in the HVAC system to its corresponding building usage. The co-simulation model can provide a reliable database to evaluate the FDD algorithm under specific building operation. Four faults scenarios and normal operation scenario are developed and tested in the quantitative system model under $10 \%$ and $20 \%$ building operation uncertainty levels. The results show that an FDD algorithm may have different performance under different building usage condition. Therefore, it is not sufficient to evaluate the FDD algorithm based on a single standard database. Standing on this point of view, to use a quantitative co-simulation model to generate the database to evaluate FDD algorithm can not only provide the clear labelled data, but also capture the variation of building usage.

\section{Acknowledgement}

This project is funded by Siemens Building Technologies Division.

\section{References}

Du, Z., Jin, X., \& Wu, L. (2007). Fault detection and diagnosis based on improved PCA with JAA method in VAV systems. Building and Environment.
Han, H., Cao, Z., Gu, B., \& Ren, N. (2010). PCA-SVMBased Automated Fault Detection and Diagnosis (AFDD) for Vapor-Compression Refrigeration Systems. HVAC\&R Research.

Katipamula, S., \& Brambley, M. R. (2005). Review Article: Methods for Fault Detection, Diagnostics, and Prognostics for Building Systems-A Review, Part I. $H V A C \& R$ Research.

Kim, W., \& Katipamula, S. (2017). a review of fault detection and diagnostics methods for building systems. Science and technology for the built environment.

Li, Z. (2012). Adaptable, scalable, probabilistic fault detection and diagnostic methods for the HVAC secondary system.

Wang, Q. (2016). ACCURACY, VALIDITY AND RELEVANCE OF PROBABILISTIC BUILDING ENERGY MODELS.

Wang, S., \& Cui, J. (2005). Sensor-fault detection, diagnosis and estimation for centrifugal chiller systems using principal-component analysis method. Applied Energy.

Wang, S., \& Xiao, F. (2004). AHU sensor fault diagnosis using principal component analysis method. Energy and Buildings.

Wang, Z., Wang, Z., He, S., Gu, X., Yan, \& ZengFeng. (2017). Fault detection and diagnosis of chillers using Bayesian network merged distance rejection and multi-source non-sensor information. Applied Energy.

Xiao, F., Wang, S., \& Zhang, J. (2006). A diagnostic tool for online sensor health monitoring in air-conditioning systems. Automation in Construction.

Xiao, F., Zhao, Y., Wen, J., \& Wang, S. (2014). Bayesian network based FDD strategy for variable air volume terminals. Automation in Construction.

Yan, K., Zhong, C., Ji, Z., \& Huang, J. (2018). Semisupervised learning for early detection and diagnosis of various air handling unit faults. Energy and Buildings.

Yang Zhao, S. W. (2013). Pattern recognition-based chillers fault detection method using Support Vector Data Description (SVDD). Applied Energy.

Zhao, Y., Xiao, F., Wen, J., Lu, Y., \& Wang, S. (2014). A robust pattern recognition-based fault detection and diagnosis (FDD) method for chillers. $H V A C \& R$ Research. 


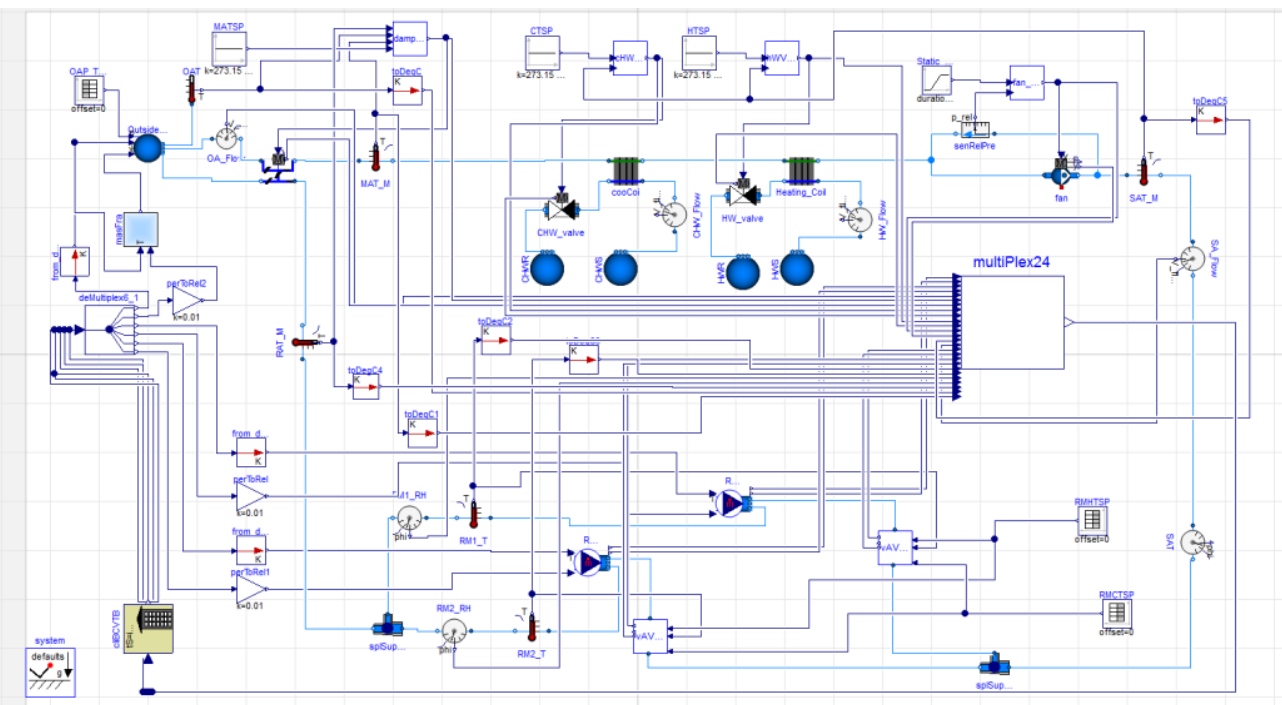

Figure 23: HVAC model in Dymola. 